\section{Precise positioning of tachistoscopic stimuli with Scientific Prototype slide changers}

\author{
JACK A. ALFORD, JR. \\ University of Texas, Austin, Texas 78712
}

An easily made device for eliminating horizontal movement of stimuli in Scientific Prototype slide changers is described. This device does not permanently alter the tachistoscope and does not interfere with normal operation of the slide changer.

The automatic slide changers of Scientific Prototype tachistoscopes, while convenient to use, exhibit considerable variability in the horizontal positioning of stimuli. This variability may become unacceptable when stimuli in two fields must be superimposed or when binocular fusion is critical. The reason for this position variability is as follows: As a slide is pushed into the projection field by the pusher arm, upper and lower spring-loaded tracks part to accomodate its height, while maintaining some pressure on the slide mount. It is this vertical pressure, rather than any horizontal barrier, which stops the slide after the pusher arm comes to a halt. Since neither the vertical nor the horizontal dimension is highly constrained in this system, mounts of varying sizes can be accommodated, a distinct advantage for most projectors incorporating this changer

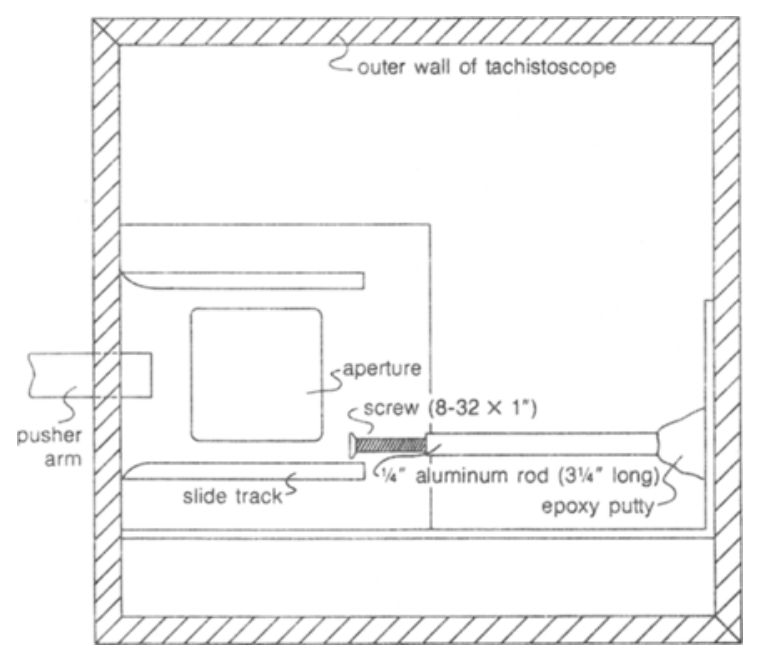

Figure 1. Schematic view of stop installed in slide changer. mechanism. However, the momentum imparted to each slide and the frictional force acting on it are not constant, so each slide moves away from the pusher arm a variable distance before coming to rest. This results in noticeable variation in what the observer sees from trial to trial, a problem in some experimental situations.

The solution to this problem is to install a stop or bumper in the slide track to insure that the slide cannot move away from the pusher arm at all, but will be reliably positioned at the same place on each trial. The installation of such a device is feasible because slide mounts used in the laboratory will generally be of the same type, and thus have the same dimensions, at least within a single experimental session.

The simple device shown in Figure 1 has several desirable properties. First, it limits the extent of slide travel without interfering with normal operation of the slide changer. Second, it is easily and cheaply manufactured in any machine shop. Third, it can be installed quickly with no permanent alteration of the tachistoscope. Finally, it can be adjusted to accommodate varying sizes of slide mounts, by inserting a long screwdriver and turning the screw one way or the other. The stop is mounted on the side wall of the optical box, projecting into the slide gate and resting on the lower slide track, using epoxy ribbon putty. When the slide enters the gate, its lower leading corner comes to rest against the screw head (which has been ground to a smaller diameter to minimize interference with the changer mechanism). If even more precise positioning is required, there is room for a second stop, parallel to the first, which stops the upper leading corner, although the lower stop alone has been found to be quite satisfactory.

The aluminum rod and machine screws are standard machine-shop items, while epoxy ribbon is available at hardware stores. This material is quite easy to apply, being thick enough to be worked like clay; it holds the rod in place without the need for other support and becomes very hard within several hours. Heat will break down the epoxy should it ever be necessary to remove the stop. A locknut may also be installed on the screw to prevent its movement, although if the machinist is instructed to make the threads in the rod fairly tight, a locknut is not required.

(Received for publication June 28, 1977; accepted July 25,1977 .) 